\title{
RESEARCH
}

Open Access

\section{Early prediction of high flow nasal cannula therapy outcomes using a modified ROX index incorporating heart rate}

Ken Junyang Goh ${ }^{1 *}$ D, Hui Zhong Chai ${ }^{1}$, Thun How Ong ${ }^{1,2}$, Duu Wen Sewa ${ }^{1}$, Ghee Chee Phua ${ }^{1,2}$ and Qiao Li Tan ${ }^{1}$

\begin{abstract}
Background: The ROX index (ratio of pulse oximetry/ $/ \mathrm{FIO}_{2}$ to respiratory rate) has been validated to predict high flow nasal cannula therapy (HFNC) outcomes in patients with pneumonia. We evaluated a modified ROX index incorporating heart rate (HR) in patients initiated on HFNC for acute hypoxemic respiratory failure and as a preventative treatment following planned extubation.

Methods: We performed a prospective observational cohort study of 145 patients treated with HFNC. ROX-HR index was defined as the ratio of ROX index over HR (beats/min), multiplied by a factor of 100. Evaluation was performed using area under the receiving operating characteristic curve (AUROC) and cutoffs assessed for prediction of HFNC failure: defined as the need for mechanical ventilation.

Results: Ninety-nine (68.3\%) and 46 (31.7\%) patients were initiated on HFNC for acute hypoxemic respiratory failure and following a planned extubation, respectively. The majority (86.9\%) of patients had pneumonia as a primary diagnosis, and 85 (56.6\%) patients were immunocompromised. Sixty-one (42.1\%) patients required intubation (HFNC failure). Amongst patients on HFNC for acute respiratory failure, HFNC failure was associated with a lower ROX and ROX-HR index recorded at time points between 1 and $48 \mathrm{~h}$. Within the first $12 \mathrm{~h}$, both indices performed with the highest AUROC at $10 \mathrm{~h}$ as follows: 0.723 (95\% Cl 0.605-0.840) and 0.739 (95\% Cl 0.626-0.853) for the ROX and ROX-HR index respectively. A ROX-HR index of $>6.80$ was significantly associated with a lower risk of HFNC failure (hazard ratio 0.301 (95\% Cl 0.143-0.663)) at $10 \mathrm{~h}$. This association was also observed at 2, 6, 18, and 24h, even with correction for potential confounding factors. For HFNC initiated post-extubation, only the ROX-HR index remained significantly associated with HFNC failure at all recorded time points between 1 and $24 \mathrm{~h}$. A ROX-HR $>8.00$ at $10 \mathrm{~h}$ was significantly associated with a lower risk of HFNC failure (hazard ratio 0.176 (95\% Cl 0.051-0.604)).
\end{abstract}

Conclusion: While validation studies are required, the ROX-HR index appears to be a promising tool for early identification of treatment failure in patients initiated on HFNC for acute hypoxemic respiratory failure or as a preventative treatment after a planned extubation.

Keywords: High flow nasal cannula, High flow oxygen therapy, Acute respiratory failure, Postextubation, Pneumonia

\footnotetext{
*Correspondence: ken.goh.junyang@singhealth.com.sg

'Department of Respiratory and Critical Care Medicine, Singapore General Hospital, 20 College Road, Singapore 169856, Singapore

Full list of author information is available at the end of the article
}

\section{$\triangle B M C$}

(c) The Author(s). 2020 Open Access This article is licensed under a Creative Commons Attribution 4.0 International License, which permits use, sharing, adaptation, distribution and reproduction in any medium or format, as long as you give appropriate credit to the original author(s) and the source, provide a link to the Creative Commons licence, and indicate if changes were made. The images or other third party material in this article are included in the article's Creative Commons licence, unless indicated otherwise in a credit line to the material. If material is not included in the article's Creative Commons licence and your intended use is not permitted by statutory regulation or exceeds the permitted use, you will need to obtain permission directly from the copyright holder. To view a copy of this licence, visit http://creativecommons.org/licenses/by/4.0/ The Creative Commons Public Domain Dedication waiver (http://creativecommons.org/publicdomain/zero/1.0/) applies to the data made available in this article, unless otherwise stated in a credit line to the data. 


\section{Introduction}

There is an increasing use of high flow nasal cannula oxygen therapy (HFNC) for acute hypoxemic respiratory failure, encouraged by evidence suggesting reduced intubation rates and possibly lower mortality [1, 2]. It is an attractive alternative to non-invasive ventilation (NIV) or conventional oxygen therapy because of its reported advantages in patient comfort, improved oxygenation, and decreased work of breathing in respiratory failure [3-5]. HFNC may also be used to reduce the rate of respiratory failure following a planned extubation [6-9]. Along with the growing use of HFNC, there is a need to improve early prediction of HFNC failure as delayed intubation is associated with increased mortality [10-12]. Patients identified as having a high risk of HFNC failure should be closely monitored or considered for early intubation, which may potentially improve patient outcomes.

The ROX (respiratory rate oxygenation) index, a ratio of pulse oximetry/fraction of inspired oxygen (P/F ratio) to respiratory rate per minute, has been validated to predict HFNC success in patients with pneumonia and acute respiratory failure $[13,14]$. It is easily derived from commonly recorded variables measured in a noninvasive manner. However, it remains to be seen if the ROX index will perform as well in patients with respiratory failure from other causes than pneumonia, or in patients with HFNC initiated after a planned extubation. In addition, it is also unclear if the ROX index can be further improved by incorporating other vital sign parameters. Tachycardia recorded as early as $1 \mathrm{~h}$ into HFNC therapy has been found to be associated with HFNC failure [15]. Heart rate is a commonly measured vital sign, and incorporation into the ROX index may improve the diagnostic accuracy of the index.

In this study, we aim to evaluate the ROX index and a modified ROX index incorporating HR, in patients initiated on HFNC for hypoxemic respiratory failure and as a preventative treatment following extubation. As heart rate has an inverse relation to HFNC success, we defined the ROX-HR (respiratory rate oxygenation-heart rate) index as the ratio of ROX index over HR (beats/min) and multiplying by a factor of 100 (Figure S1).

\section{Methods}

\section{Study design}

We performed a prospective observational cohort study of patients initiated on HFNC (Optiflow device-MR850 heated humidified, RT202 delivery tubing and nasal cannula; Fisher \& Paykel Healthcare, Auckland, New Zealand) ${ }^{\mathrm{TM}}$ at a medical intensive care unit (ICU) and intermediate care area of a tertiary-care medical centre. All consecutive patients initiated on HFNC from February 2017 to September 2019 were recruited for the study. Exclusion criteria of the study were patients initiated on
HFNC for bronchoscopic procedures or as a rescue therapy post-extubation, patients who were started on noninvasive ventilation after HFNC failure, and patients with a 'do not resuscitate or intubate' order. We obtained approval from our institutional review board for this study (CIRB Ref 2016/2988). No written consent was required in view of the purely observational nature of the study.

\section{HFNC protocol and management}

Patients eligible for HFNC in our centre's protocol include patients with acute hypoxemic respiratory failure, defined as having a respiratory rate $>25$ breaths $/$ min and a P/F ratio of $<300 \mathrm{mmHg}$ on an oxygen device delivering $10 \geq$ litres/min (LPM), in the absence of chronic respiratory failure. In our centre, HFNC may also be initiated as a preventative treatment, initiated immediately following a planned extubation. Prior to extubation, all patients had to fulfil clinical weaning criteria with a successful spontaneous breathing trial. Protocol exclusion criteria for all patients are the presence of hypercapnia $\left(\mathrm{PaCO}_{2}>\right.$ $45 \mathrm{mmHg}$ ), acute respiratory failure secondary to asthma, chronic obstructive pulmonary disease (COPD) exacerbation or cardiogenic pulmonary edema, hemodynamic instability requiring vasopressor support, Glasgow coma scale $(\mathrm{GCS})<12$ and epistaxis or recent facial or nasal surgery. HFNC was initiated at a minimum initial flow of $40 \mathrm{LPM}$. Flows were increased up to $60 \mathrm{LPM}$ if required, or $\mathrm{FIO}_{2}$ adjusted as appropriate, with a target $\mathrm{SpO}_{2}$ of $\geq 92 \%$. Discontinuation of HFNC and initiation of intubation and mechanical ventilation were based on the clinical judgement of the primary physician, guided by a protocol recommendation to consider mechanical ventilation in the presence of persistent/worsening respiratory distress, respiratory rate $>40$ breaths $/ \mathrm{min}, \mathrm{SpO}_{2}<90 \%$ for more than 5 min despite maximum flow and $\mathrm{FIO}_{2}$, acidemia with $\mathrm{pH}<7.35$, significant hemodynamic instability (defined as systolic blood pressure $<90 \mathrm{mmHg}$, mean arterial pressure $<65 \mathrm{mmHg}$ or vasopressor requirement), deterioration in neurological status $(\mathrm{GCS}<12)$ or inability to clear secretions.

\section{Data collection}

Patient demographics, Charlson comorbidity index (CCI), clinical severity scores and arterial blood gas sampling before initiation of HFNC were recorded upon inclusion into the study. Chest radiographs (CXR) were evaluated at the beginning of HFNC therapy. The acute physiologic assessment and chronic health evaluation II (APACHE II) score and sequential organ failure assessment score (SOFA) were recorded based on the highest scores in the $24 \mathrm{~h}$ preceding HFNC initiation $[16,17]$. The presence of chronic kidney disease (CKD) was defined as having a baseline serum creatinine of $>265 \mu \mathrm{mol} / \mathrm{L}$ or requiring long-term dialysis. Chronic pulmonary disease 
was defined as symptomatic dyspnea from a chronic respiratory condition such as COPD or interstitial lung disease. Patients were considered as immunocompromised if they had one or more of the following: haematological or solid organ malignancy, prior haematological or solid organ transplantation, human immunodeficiency (HIV) infection, liver cirrhosis with portal hypertension or receiving long-term immunosuppressive therapy. Patients were followed up until in-hospital death or discharge from hospital.

\section{ROX and ROX-HR index}

We recorded the HR and ROX index before initiation of HFNC and at 1, 2, 4, 6, 8, 10,12, 18, 24 and $48 \mathrm{~h}$ after HFNC initiation. HFNC success was defined by liberation of HFNC, and failure was defined by intubation and mechanical ventilation. Duration of HFNC was recorded as the time (h) from initiation of HFNC to successful liberation or failure. At the time of termination of HFNC, the ROX and ROX-HR index based on the latest available parameters from $1 \mathrm{~h}$ before termination were also recorded.

\section{Statistical analysis}

Data are presented as number (\%) for categorical variables and median (interquartile range [IQR]) for continuous variables. Data and analyses are separated based on the indication for HFNC: acute respiratory failure vs post-extubation. Patients with and without successful HFNC were compared with respect to clinical and demographic characteristics by using the Mann-Whitney $U$ test for continuous variables and the chi-square test or Fisher exact test as appropriate for categorical variables. The ROX and ROX-HR index at different time points were evaluated with the area under the receiver operating characteristic curve (AUROC) for the ability to correctly classify patients as HFNC success or failure. Cutoffs for the ROX and ROX-HR index, rounded off to the nearest 0.1 , were chosen to maximise the sum of sensitivity and specificity based on the receiving operating characteristic curves. From these cutoffs, KaplanMeier (KM) plots for HFNC failure were determined and compared using the log-rank test. Univariate and multivariate Cox proportional regression analysis was performed to evaluate the hazard ratio for cumulative probability of HFNC failure based on the ROX and ROX-HR index at different time points. Covariates that were associated with HFNC failure ( $p$ value of $<0.10$ ) on univariate Cox proportional regression analysis were included in the multivariate analysis. Statistical difference was considered significant at $\mathrm{p} \leq 0.05$. All statistical analyses were performed using the SPSS software (IBM SPSS Statistics ver. 22 Chicago, IL, USA).

\section{Results}

Patient population and HFNC outcomes

One hundred and forty-five patients were included in the study analysis. Nineteen patients were excluded: six patients had HFNC support initiated for bronchoscopy, five patients were switched from HFNC to NIV therapy, one patient had HFNC terminated due to epistaxis, one patient had HFNC terminated for transfer to the operating theatre for surgery, and six patients had a 'do not resuscitate or intubate' order. Immunocompromised patients made up 56.6\% $(n=82)$ of the study population. Twenty patients had recently received chemotherapy for solid organ malignancies, 28 patients had an underlying haematological malignancy or a bone marrow transplant, and 29 patients were receiving chronic immunosuppressive therapy.

Ninety-nine patients $(68.3 \%)$ were initiated on HFNC for acute hypoxemic respiratory failure (Table 1). Pneumonia was the most common primary diagnosis $(87 / 99$, 87.9\%). The median P/F ratio was 94 (IQR 74-138), and SOFA score was 4 (IQR 3-6) at the time of HFNC initiation. Forty-five (45.5\%) patients required intubation (HFNC failure) at a median of 16 (IQR 7-36) h after HFNC initiation. HFNC failure was associated with a higher SOFA and APACHE II score (recorded as the highest score in the preceding $24 \mathrm{~h}$ before initiation of HFNC) and an increased proportion of CXR quadrants affected at the time of HFNC initiation (Table 1). There were no significant differences found in the proportion of immunocompromised patients or pre-HFNC arterial blood gas analysis ( $\mathrm{pH}, \mathrm{P} / \mathrm{F}$ ratio, $\mathrm{PaCO}_{2}$ and serum bicarbonate).

Forty-six patients (31.7\%) were initiated on HFNC post-extubation (Table 2). The median duration of mechanical ventilation prior to extubation was 114 (IQR 61-194) h. Prior to extubation, the median $\mathrm{pH}$ was 7.45 (IQR 7.42-7.48), with a $\mathrm{PaCO}_{2}$ of 41 (IQR 36-44) $\mathrm{mmHg}$ and P/F ratio of 164 (IQR 137-184). Sixteen (16/ $46,34.8 \%)$ patients required re-intubation, at a median duration of 46 (10-87) h after HFNC initiation. Patients with HFNC failure were more likely to be immunocompromised ( $75 \%$ vs $37 \%, p=0.029)$.

In both groups of patients (acute respiratory failure and post-extubation), HFNC failure was associated with a higher hospital and ICU mortality rate (Tables 1 and 2). Among all patients with HFNC failure, 22 (22/61, 36.1\%) and $38(38 / 61,62.3 \%)$ patients were initiated on mechanical ventilation within 12 and $24 \mathrm{~h}$, respectively. Initiation of mechanical ventilation after $24 \mathrm{~h}$ of HFNC was associated with a higher in-hospital $(78.3 \%$ vs $50.0 \%, p=0.029)$ and ICU mortality rate $(69.6 \%$ vs $35.1 \%, p=0.009)$. For the 61 patients with HFNC failure, the Kaplan-Meier plot for the probability of being free of mechanical ventilation is shown in Fig. 1a and b, which illustrates that patients who did not survive hospital admission had a longer duration of HFNC before intubation. 
Table 1 Comparing baseline characteristics and outcomes of patients with acute hypoxemic respiratory failure $(n=99)$

\begin{tabular}{|c|c|c|c|}
\hline & HFNC success $(n=54)$ & HFNC failure $(n=45)$ & $p$ value \\
\hline Age, years & $65(56-72)$ & $63(55-70)$ & 0.171 \\
\hline Male gender & $36(66.7)$ & $20(44.4)$ & 0.026 \\
\hline Charlson comorbidity index & $5(3-7)$ & $4(2-6)$ & 0.086 \\
\hline Moderate to severe CKD & $12(22.2)$ & $5(11.1)$ & 0.144 \\
\hline Congestive heart failure & $0(0.0)$ & $2(4.4)$ & 0.204 \\
\hline Chronic respiratory disease & $3(10.0)$ & $2(7.7)$ & 1.000 \\
\hline Immunocompromised host & $35(64.8)$ & $24(53.3)$ & 0.246 \\
\hline Solid organ cancer with chemotherapy & $8(22.9)$ & $7(29.2)$ & 0.585 \\
\hline Hematological transplant or malignancy & $11(31.4)$ & $7(29.2)$ & 0.853 \\
\hline Chronic immunosuppressive therapy & $13(37.1)$ & $9(37.5)$ & 0.978 \\
\hline HIV/AIDS & $3(8.6)$ & $1(4.2)$ & 0.639 \\
\hline APACHE $\|^{*}$ & $16(12-21)$ & $19(15-23)$ & 0.011 \\
\hline SOFA* & $4(3-6)$ & $5(4-7)$ & 0.010 \\
\hline \multicolumn{4}{|l|}{ Primary etiology for respiratory failure } \\
\hline Pneumonia & $47(87.0)$ & $40(88.9)$ & 0.779 \\
\hline nterstitial lung disease/drug induced pneumonitis & $0(0.0)$ & $3(6.7)$ & 0.090 \\
\hline Cancer/Lymphangitis carcinomatosis & $2(3.7)$ & $2(4.4)$ & 1.000 \\
\hline Others & $5(9.3)$ & $0(0.0)$ & 0.061 \\
\hline Vasopressor support at time of HFNC initiation & $1(1.9)$ & $0(0.0)$ & 1.000 \\
\hline Number of quadrants affected on CXR & $3(2-4)$ & $4(3-4)$ & 0.013 \\
\hline \multicolumn{4}{|l|}{ Arterial blood gas analysis pre-HFNC initiation } \\
\hline $\mathrm{pH}$ & $7.43(7.39-7.47)$ & $7.44(7.39-7.47)$ & 0.754 \\
\hline $\mathrm{PaO}_{2} / \mathrm{FIO}_{2}$ ratio & $94(72-139)$ & $92(74-139)$ & 0.697 \\
\hline $\mathrm{PaCO}_{2}, \mathrm{mmHg}$ & $36(32-40)$ & $34(30-39)$ & 0.055 \\
\hline Serum bicarbonate, $\mu \mathrm{mol} / \mathrm{L}$ & $24(22-26)$ & $23(22-25)$ & 0.341 \\
\hline Duration of HFNC, $h$ & $41.5(22.1-70.1)$ & $16.2(7.4-35.5)$ & $<0.001$ \\
\hline Max $\mathrm{FIO}_{2}$ on $\mathrm{HFNC}$ & $80(70-100)$ & $100(80-100)$ & 0.044 \\
\hline Max flow on HFNC, L/min & $50(40-60)$ & $60(50-60)$ & 0.084 \\
\hline Hospital mortality & $11(20.4)$ & $27(60.0)$ & $<0.001$ \\
\hline ICU mortality & $7(15.2)$ & $21(47.7)$ & 0.001 \\
\hline
\end{tabular}

Values are expressed in number (percentage) and median (interquartile range). HFNC high flow nasal cannula, CKD chronic kidney disease, HIV human immunodeficiency, AIDS acquired immunodeficiency syndrome, APACHE acute physiologic assessment and chronic health evaluation, SOFA sequential organ failure assessment score, CXR chest x-ray, ICU intensive care unit. Etiology for 'Others' include diffuse alveolar haemorrhage, pulmonary embolism and cardiogenic pulmonary edema.

${ }^{*} \mathrm{APACHE}$ II and SOFA scores were recorded based on the highest scores in the $24 \mathrm{~h}$ preceding HFNC initiation

\section{Performance of the ROX and ROX-HR index}

In patients initiated on HFNC for acute respiratory failure, after 2, 6, 10 and $24 \mathrm{~h}, 98$ (99.0\%), 90 (90.9\%), 83 (83.9\%) and $67(67.7 \%)$ patients remained free of mechanical ventilation, respectively (Table 3 ). Patients with HFNC failure had a significantly lower ROX and ROX$\mathrm{HR}$ index recorded at all time points, and a significantly higher heart rate was observed at 1, 2, 4, 10 and $12 \mathrm{~h}$ of HFNC (Table 3). Within the first $12 \mathrm{~h}$, both indices appeared to have the highest diagnostic accuracy at $10 \mathrm{~h}$ with an AUROC of 0.723 (95\% confidence interval (CI) $0.605-0.862$ ) and 0.739 (95\% CI 0.626-0.853) for the
ROX index and ROX-HR index, respectively. Figure 2 illustrates the proportion of patient with successful HFNC for acute respiratory failure, based on ROX-HR index scores at 2 and $10 \mathrm{~h}$.

In patients initiated on HFNC post-extubation, 45 (97.8\%), 43 (93.5\%), 42 (91.3\%) and 40 (87.0\%) patients remained free of mechanical ventilation at 2, 6, 10 and $24 \mathrm{~h}$, respectively (Table 4). Apart from the 48-h time point, patients with HFNC failure had a consistently lower ROX-HR, while no significant difference was seen with the ROX index measured at 2, 4, 8 and $18 \mathrm{~h}$ (Table 4). Heart rate alone predicted HFNC outcomes with an 
Table 2 Comparing baseline characteristics and outcomes of patients initiated on HFNC post extubation $(n=46)$

\begin{tabular}{|c|c|c|c|}
\hline & HFNC success $(n=30)$ & HFNC failure $(n=16)$ & $p$ value \\
\hline Age, years & $64(53-71)$ & $61(52-72)$ & 0.982 \\
\hline Male gender & $17(56.7)$ & $7(43.8)$ & 0.978 \\
\hline Charlson comorbidity index & $5(3-6)$ & $4(3-6)$ & 0.557 \\
\hline Moderate to severe CKD & $4(13.3)$ & $2(12.5)$ & 1.000 \\
\hline Congestive heart failure & $1(3.3)$ & $2(12.5)$ & 0.274 \\
\hline Chronic respiratory disease & $3(21.4)$ & $1(8.3)$ & 0.598 \\
\hline Immunocompromised host & $11(36.7)$ & $12(75.0)$ & 0.029 \\
\hline Solid organ cancer with chemotherapy & $4(36.4)$ & $1(8.3)$ & 0.155 \\
\hline Hematological transplant or malignancy & $6(54.5)$ & $4(33.3)$ & 0.414 \\
\hline Chronic immunosuppressive therapy & $1(9.1)$ & $6(50.0)$ & 0.069 \\
\hline HIV/AIDS & $0(0.0)$ & $1(8.3)$ & 1.000 \\
\hline APACHE $\|^{*}$ & $15(13-19)$ & $16(11-21)$ & 0.899 \\
\hline SOFA* & $5(3-9)$ & $4(3-7)$ & 0.368 \\
\hline \multicolumn{4}{|l|}{ Primary etiology for respiratory failure } \\
\hline Pneumonia & $25(83.3)$ & $14(87.5)$ & 1.000 \\
\hline Interstitial lung disease/drug induced pneumonitis & $3(10.0)$ & $0(0.0)$ & 0.542 \\
\hline Cancer/Lymphangitis carcinomatosis & $0(0.0)$ & $2(12.5)$ & 0.116 \\
\hline Others & $2(6.7)$ & $0(0.0)$ & 0.536 \\
\hline Duration of mechanical ventilation before extubation, $\mathrm{h}$ & $94(53-197)$ & $171(92-194)$ & 0.137 \\
\hline Vasopressor support at time of HFNC initiation & $0(0.0)$ & $0(0.0)$ & NA \\
\hline Number of quadrants affected on CXR & $3(3-4)$ & $4(3-4)$ & 0.327 \\
\hline \multicolumn{4}{|l|}{ Arterial blood gas analysis pre-HFNC initiation } \\
\hline $\mathrm{pH}$ & $7.46(7.43-7.48)$ & $7.45(7.41-7.48)$ & 0.406 \\
\hline $\mathrm{PaO}_{2} / \mathrm{FIO}_{2}$ ratio & 165 153-190) & $157(129-180)$ & 0.122 \\
\hline $\mathrm{PaCO}_{2}, \mathrm{mmHg}$ & $41(36-44)$ & $41(38-45)$ & 0.773 \\
\hline Serum bicarbonate, $\mu \mathrm{mol} / \mathrm{L}$ & $27(24-30)$ & $27(24-31)$ & 0.936 \\
\hline Duration of HFNC, $h$ & $29.3(22.6-49.8)$ & $46.0(9.6-86.6)$ & 0.827 \\
\hline Max $\mathrm{FIO}_{2}$ on $\mathrm{HFNC}$ & $50(50-60)$ & $80(60-100)$ & $<0.001$ \\
\hline Max flow on HFNC, L/min & $50(40-50)$ & $60(50-60)$ & 0.021 \\
\hline Hospital mortality & $6(20.0)$ & $10(62.5)$ & 0.004 \\
\hline ICU mortality & $4(13.3)$ & $8(50.0)$ & 0.013 \\
\hline
\end{tabular}

Values are expressed in number (percentage) and median (interquartile range). HFNC high flow nasal cannula, CKD chronic kidney disease, HIV human immunodeficiency, AIDS acquired immunodeficiency syndrome, APACHE acute physiologic assessment and chronic health evaluation, SOFA sequential organ failure assessment score, CXR chest $\mathrm{x}$-ray, ICU intensive care unit. Etiology for 'Others' include diffuse alveolar haemorrhage, pulmonary embolism and cardiogenic pulmonary edema.

${ }^{*}$ APACHE II and SOFA scores were recorded based on the highest scores in the $24 \mathrm{~h}$ preceding HFNC initiation

AUROC of 0.693 (95\% CI 0.529-0.856) and 0.699 (95\% CI $0.518-0.881)$ at 2 and $4 \mathrm{~h}$, respectively. Within the first 12 $\mathrm{h}$, the highest AUROC was found with the ROX index (0.773, 95\% CI 0.617-0.928) and the ROX-HR index (0.804, 95\% CI $0.660-0.948)$ at $10 \mathrm{~h}$ of HFNC therapy. Figure S2 illustrates the proportion of patient with successful HFNC after a planned extubation, based on ROX-HR index scores at 2 and $10 \mathrm{~h}$.

In all patients, HFNC success was associated with a significantly higher increase in ROX-HR index from the
2 to $10 \mathrm{~h}$ and 6 to $10 \mathrm{~h}$ time points-this was not observed with the ROX index (Table S1).

\section{Evaluating cutoffs of the ROX-HR and ROX index for patients with acute respiratory failure}

Using the ROC curve at $10 \mathrm{~h}$ into HFNC therapy, cutoffs for the ROX and ROX-HR were determined to be 5.80 and 6.80, respectively, for the prediction of HFNC success. The sensitivity, specificity, positive predictive value (PPV) and negative predictive values (NPV) of each 


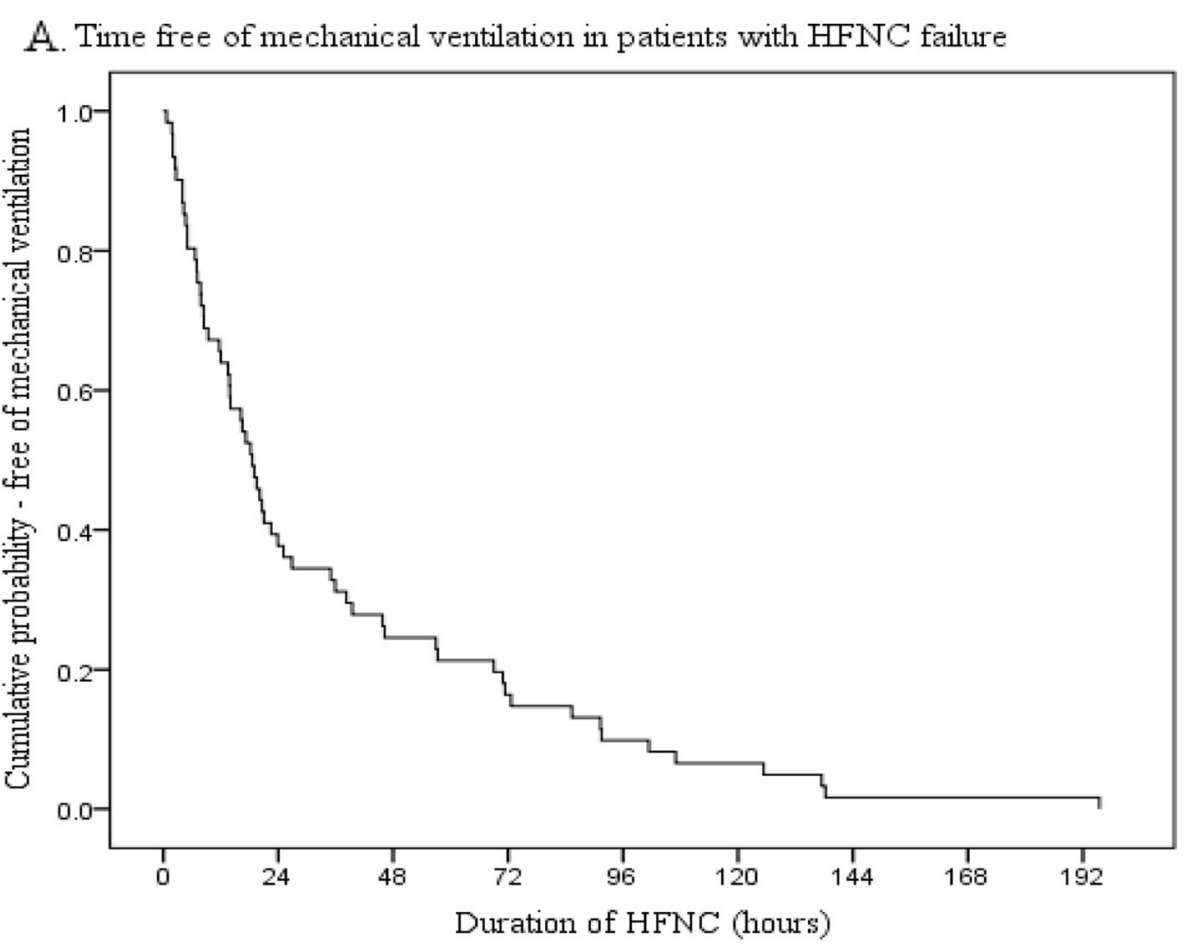

B. Time free of mechanical ventilation based on in-hospital mortality

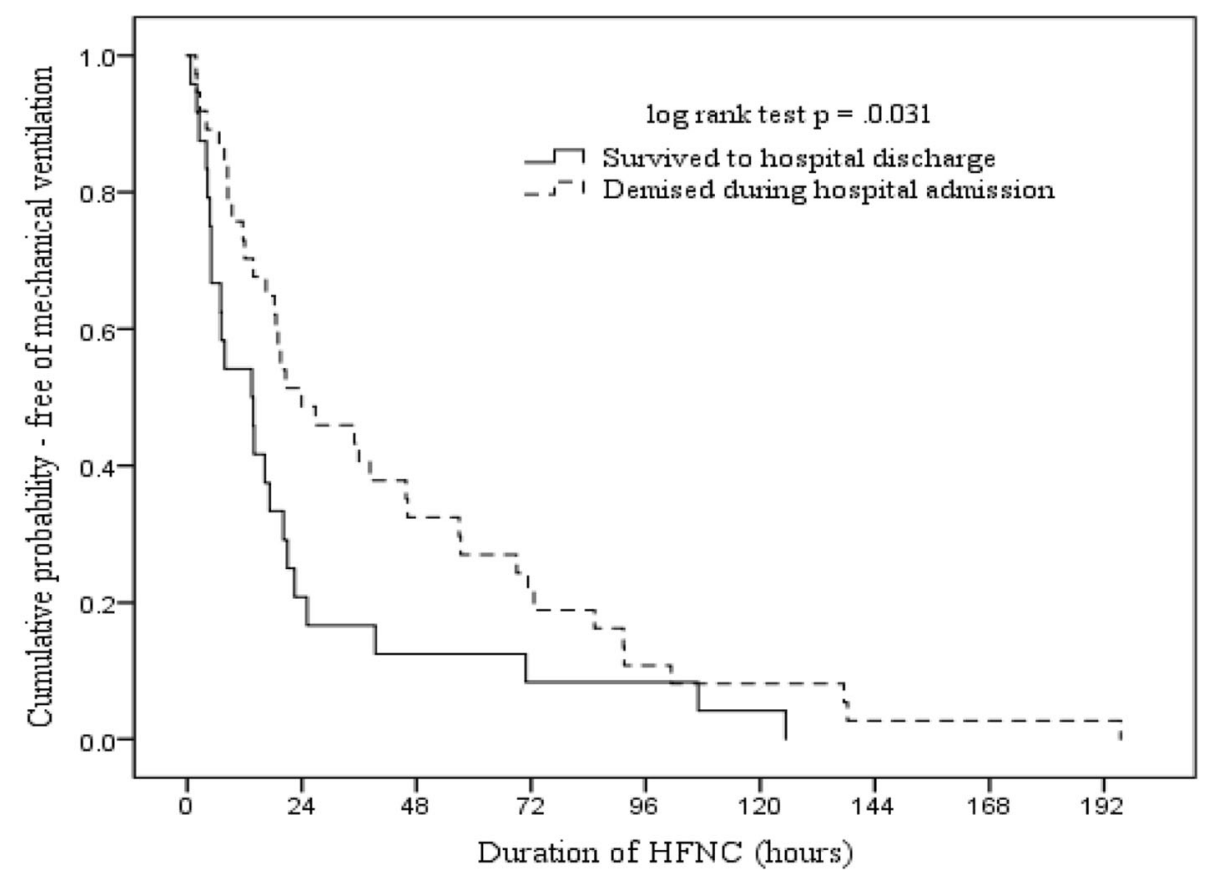

Fig. 1 a Kaplan-Meier plot for the time free of mechanical ventilation in patients with HNFC failure $(n=61)$. $\mathbf{b}$ Among patients with HFNC failure, patients who did not survive had a longer duration of HFNC before intubation and mechanical ventilation

index at 2, 6, 10, 18 and $24 \mathrm{~h}$ are summarised in Table 5. With Cox proportional regression analysis, a ROX-HR index of $>6.80$ was associated with a lower risk of HFNC failure at all time points in the first $24 \mathrm{~h}$, even after correcting for possible confounders (Gender, APACHE II score, CCI and the number of CXR quadrants involved) (Table 6). Kaplan-Meier plots illustrating significant differences in probability of HFNC success with 
Table 3 Variables and diagnostic accuracy (for HFNC outcomes) at different time points during HFNC therapy initiated for acute respiratory failure

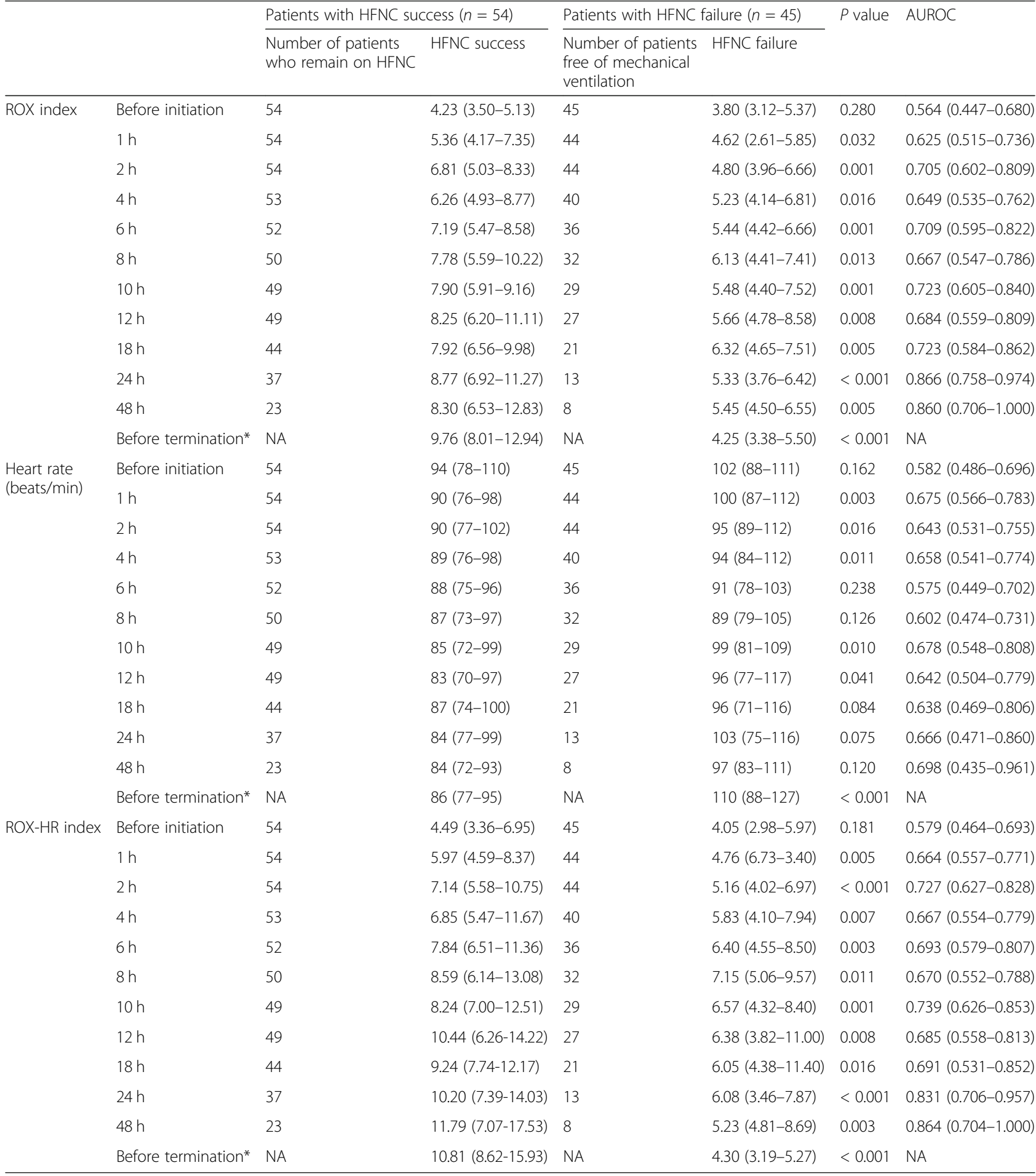

HFNC high flow nasal cannula therapy, $A U R O C$ area under the receiver operating characteristic curve, $N A$ not applicable *Successful or failed termination of HFNC

a cutoff of 6.80 for the ROX-HR index are illustrated in Fig. 3a-c. A second cutoff of ROX $<5.00$ and ROX-HR $<5.00$ was determined from the ROC curves at $10 \mathrm{~h}$, and their performance for the predictirised in Table S2. Compared to $\mathrm{ROX}<5.00$, a ROX-HR index $<5.00$ appeared to perform with higher positive and lower negative likelihood ratios at 6, 10, 18 and $24 \mathrm{~h}$. 

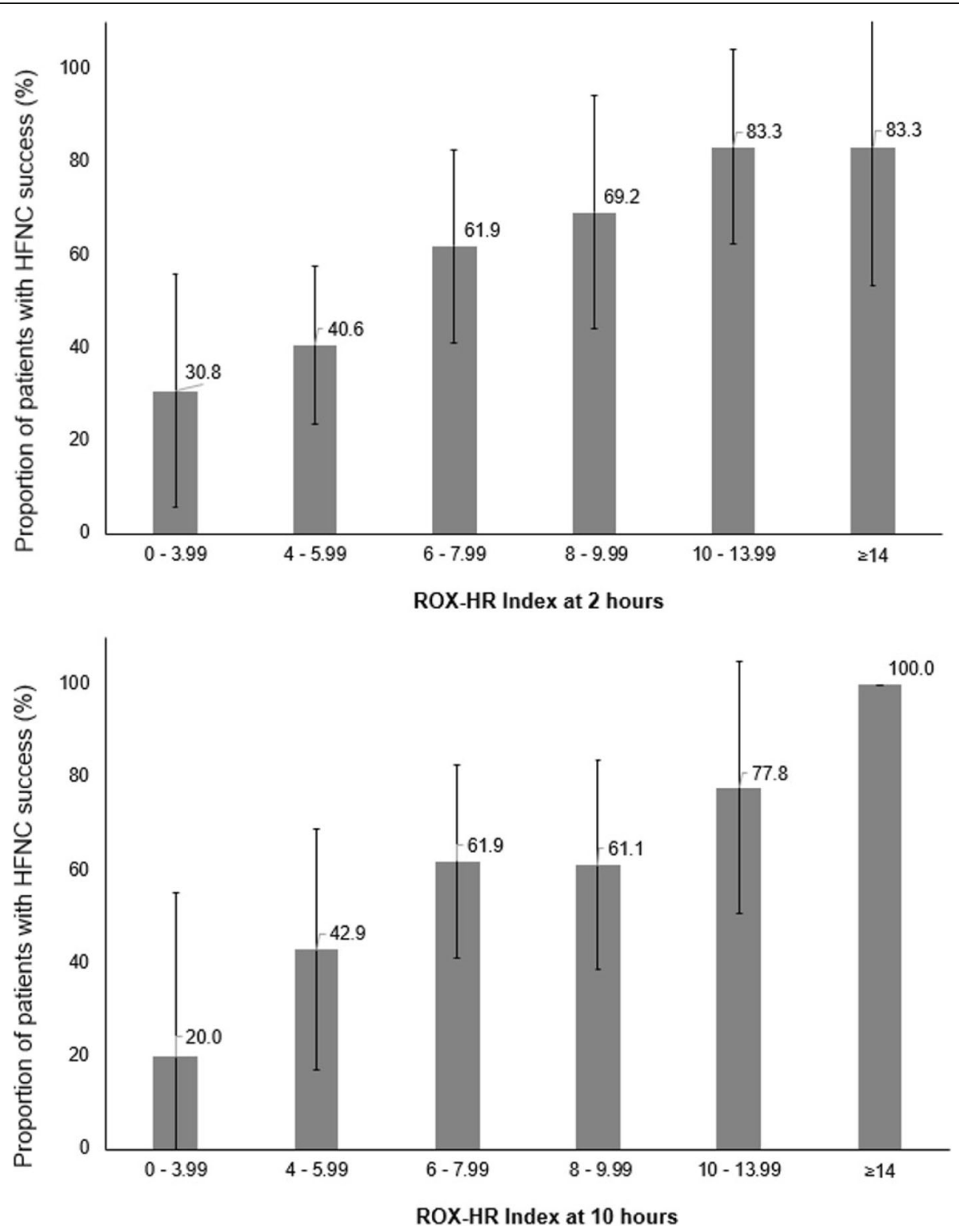

Fig. 2 Proportion of patients with successful HFNC for acute respiratory failure, based ROX-HR index at $2 \mathrm{~h}$ (top graph) and $10 \mathrm{~h}$ (bottom graph)

We evaluated the ROX index based on previously established cutoffs of 4.88 (by Roca et al.) at 2, 6 and $12 \mathrm{~h}$ (Table S3 and S4) [14]. Similarly, as with a ROX index > 5.80 , there was no significant association of a lower risk of HFNC failure with a ROX index $\geq 4.88$ at $12 \mathrm{~h}$ (Table S3). Roca et al. also reported cutoffs of 2.85, 3.47 and 3.85 at 2, 6 and $12 \mathrm{~h}$ for the ROX index for the prediction of HFNC failure [14]. We evaluated these cutoffs in our study population, while these cutoffs performed with good specificity (>95\%), the sensitivity remained poor $(<15 \%)$. In contrast, a ROX-HR index of $<4.50$ at $2 \mathrm{~h}$ and $<5.00$ at 6 and $12 \mathrm{~h}$ performed with reasonable sensitivity $(>34 \%)$ and specificity $(>88 \%)$.

\section{Evaluating cutoffs of the ROX-HR and ROX index for patients extubated to HFNC}

A cutoff of 7.00 and 8.00 was determined for the ROX and ROX-HR index, respectively, based on the ROC curves at $10 \mathrm{~h}$ into HFNC therapy. Table 5 summarises the sensitivity, specificity, PPV and NPV of the cutoffs at various time points within $24 \mathrm{~h}$ of HFNC initiation. The ROX-HR index performed with equal or higher sensitivity and specificity at 2, 6 and $10 \mathrm{~h}$ (Table 5). On univariate Cox proportional regression analysis, a ROX-HR index of $>8.00$ was significantly associated with a lower risk of HFNC failure at 6 and $10 \mathrm{~h}$, which was not seen with the 
Table 4 Variables and diagnostic accuracy (for HFNC outcomes) at different time points during HFNC therapy initiated after a planned extubation

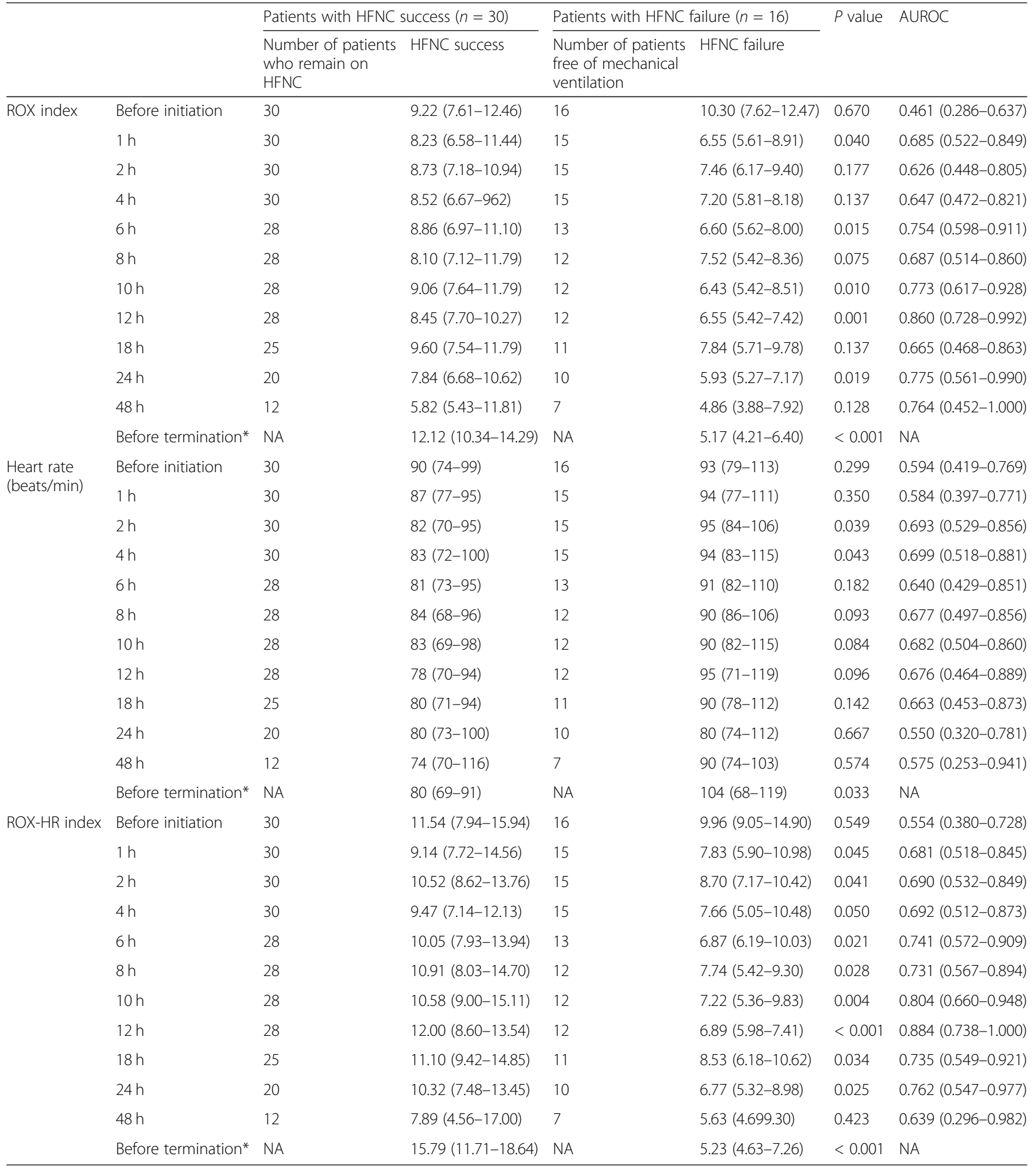

HFNC High flow nasal cannula therapy, AUROC area under the receiver operating characteristic curve, NA not applicable

*Successful or failed termination of HFNC

ROX index $>7.00$ (Table 7). Kaplan-Meier plots illustrating the probability of HFNC success with a cutoff of 8.00 for the ROX-HR index at 2, 6 and $10 \mathrm{~h}$ are shown in Fig. 3d, e. A second cutoff of 6.00 was determined from ROC curves at $10 \mathrm{~h}$, and the performance of a cutoff of ROX $<6.00$ and ROX-HR $<$ 6.00 for the prediction of HFNC failure is also summarised in Table S2. 
Table 5 Prediction of HFNC success based on ROX and ROX-HR cut offs at different time points

\begin{tabular}{|c|c|c|c|c|c|c|}
\hline & Sensitivity (\%) & Specificity (\%) & PPV (\%) & NPV (\%) & LR+ & LR- \\
\hline \multicolumn{7}{|c|}{ A. Prediction of HFNC success for patients initiated on HFNC for acute respiratory failure } \\
\hline 2-h ROX-HR > 6.80 & 55.56 & 74.41 & 73.17 & 57.14 & 2.17 & 0.60 \\
\hline 2-h ROX > 5.80 & 61.11 & 67.44 & 70.21 & 58.00 & 1.87 & 0.58 \\
\hline 6-h $\mathrm{ROX}-\mathrm{HR}>6.80$ & 70.00 & 57.14 & 70.00 & 57.14 & 1.63 & 0.53 \\
\hline 6-h ROX > 5.80 & 74.00 & 57.14 & 71.15 & 60.60 & 1.73 & 0.46 \\
\hline 10-h ROX-HR > 6.80 & 78.26 & 58.62 & 75.00 & 62.96 & 1.89 & 0.37 \\
\hline 10-h ROX > 5.80 & 78.26 & 58.62 & 75.00 & 62.96 & 1.89 & 0.37 \\
\hline 18-h ROX-HR > 6.80 & 80.00 & 55.00 & 78.05 & 57.89 & 1.78 & 0.36 \\
\hline 18-h ROX > 5.80 & 87.50 & 35.00 & 72.92 & 58.33 & 1.35 & 0.36 \\
\hline 24-h ROX-HR > 6.80 & 81.82 & 64.29 & 84.39 & 60.00 & 2.29 & 0.28 \\
\hline 24-h ROX > 5.80 & 84.85 & 57.14 & 82.35 & 61.54 & 1.98 & 0.27 \\
\hline \multicolumn{7}{|c|}{ B. Prediction of HFNC success for patients initiated on HFNC after a planned extubation } \\
\hline 2-h ROX-HR > 8.00 & 82.10 & 40.00 & 71.90 & 54.50 & 1.37 & 0.45 \\
\hline 2-h ROX > 7.00 & 78.60 & 40.00 & 71.00 & 50.00 & 1.31 & 0.54 \\
\hline 6-h ROX-HR > 8.00 & 74.10 & 63.60 & 83.30 & 50.00 & 2.04 & 0.41 \\
\hline 6-h ROX > 7.00 & 74.10 & 54.50 & 80.00 & 46.20 & 1.63 & 0.48 \\
\hline 10-h ROX-HR > 8.00 & 84.60 & 63.60 & 84.60 & 63.60 & 2.32 & 0.24 \\
\hline 10-h ROX > 7.00 & 84.60 & 54.50 & 81.50 & 60.00 & 1.86 & 0.28 \\
\hline 18-h ROX-HR > 8.00 & 82.60 & 30.00 & 73.10 & 42.90 & 1.18 & 0.58 \\
\hline 18-h ROX > 7.00 & 78.30 & 40.00 & 75.00 & 44.40 & 1.31 & 0.54 \\
\hline 24-h ROX-HR > 8.00 & 66.70 & 66.70 & 82.40 & 46.20 & 2.00 & 0.50 \\
\hline 24-h ROX > 7.00 & 71.40 & 77.80 & 88.20 & 53.80 & 3.22 & 0.37 \\
\hline
\end{tabular}

HFNC high flow nasal cannula therapy, $P P V$ positive predictive value, $N P V$ negative predictive value, $L R$ likelihood ratio

\section{Discussion}

The results of this study suggest that the ROX-HR index may be a useful tool for early prediction of HFNC outcomes. This applies to patients with acute hypoxemic respiratory failure as well as patients initiated on HFNC as a preventative treatment following a planned extubation.
It is easily derived from commonly recorded variables measured in a non-invasive manner and is a practical assessment tool that can be readily applied by the bedside.

For patients initiated on HFNC for acute hypoxemic respiratory failure, the $\mathrm{ROX}-\mathrm{HR}$ index appears to perform consistently (AUROC > 0.65) in discriminating

Table 6 Cox proportional regression analysis evaluating ROX $>5.80$ and ROX-HR $>6.80$ for the likelihood of HFNC failure in patients initiated on HFNC for acute respiratory failure

\begin{tabular}{|c|c|c|c|c|c|}
\hline & & Univariate analysis & $p$ value & Multivariate analysis & $p$ value \\
\hline \multirow[t]{5}{*}{$\mathrm{ROX}>5.80$} & $2 \mathrm{~h}$ & $0.403(0.213-0.763)$ & 0.005 & $0.460(0.238-0.892)$ & 0.021 \\
\hline & $6 \mathrm{~h}$ & $0.365(0.187-0.714)$ & 0.003 & $0.494(0.260-1.015)$ & 0.055 \\
\hline & $10 \mathrm{~h}$ & $0.299(0.142-0.626)$ & 0.001 & $0.397(0.176-0.894)$ & 0.026 \\
\hline & $18 \mathrm{~h}$ & $0.300(0.119-0.756)$ & 0.011 & $0.138(0.036-0.532)$ & 0.004 \\
\hline & $24 \mathrm{~h}$ & $0.194(0.067-0.563)$ & 0.003 & $0.338(0.101-1.136)$ & 0.079 \\
\hline \multirow[t]{5}{*}{$\mathrm{ROX}-\mathrm{HR}>6.80$} & $2 \mathrm{~h}$ & $0.353(0.178-0.702)$ & 0.003 & $0.423(0.211-0845)$ & 0.015 \\
\hline & $6 \mathrm{~h}$ & $0.394(0.201-0.772)$ & 0.007 & $0.408(0.201-0.828)$ & 0.013 \\
\hline & $10 \mathrm{~h}$ & $0.301(0.143-0.663)$ & 0.002 & $0.369(0.162-0.841)$ & 0.018 \\
\hline & $18 \mathrm{~h}$ & $0.254(0.105-0.616)$ & 0.002 & $0.252(0.098-0.645)$ & 0.004 \\
\hline & $24 \mathrm{~h}$ & $0.177(0.059-0.534)$ & 0.002 & $0.234(0.071-0.771)$ & 0.017 \\
\hline
\end{tabular}


A. Probability of HFNC success based on ROX-HR at 2 hours

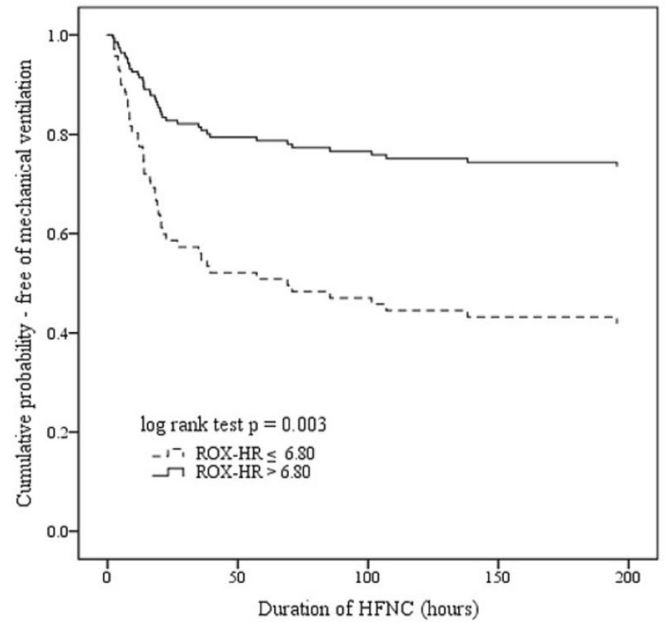

C. Probability of HFNC success based on ROX-HR at 10 hours

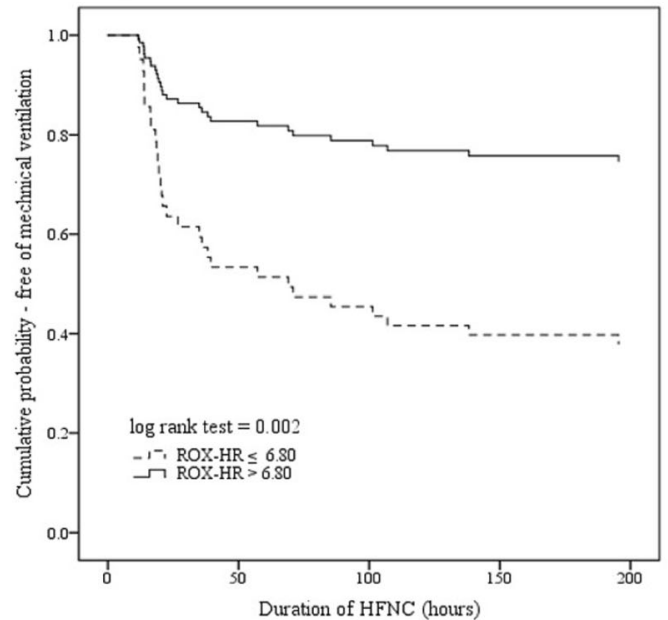

E. Probability of HFNC success based on ROX-HR at 6 hours

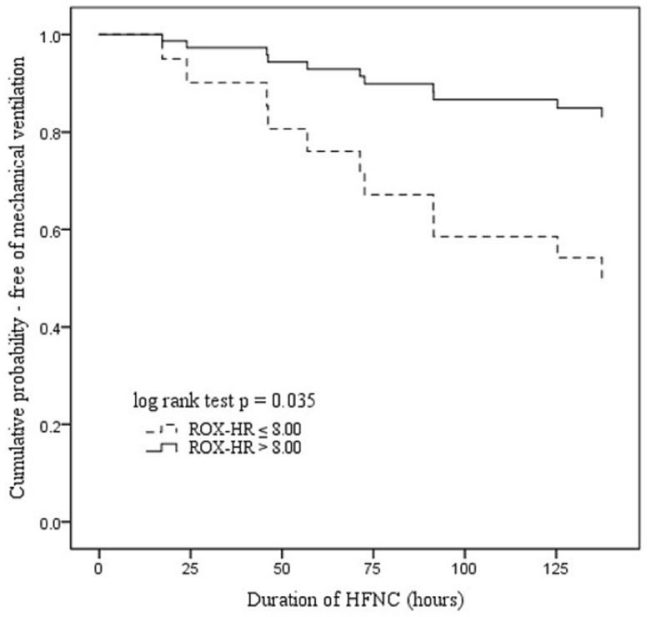

B. Probability of HFNC success based on ROX-HR at 6 hours

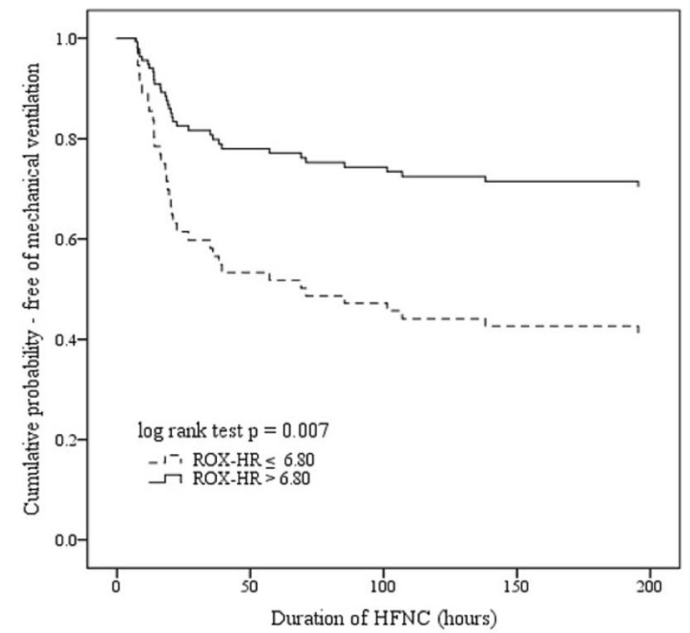

D. Probability of HFNC success based on ROX-HR at 2 hours

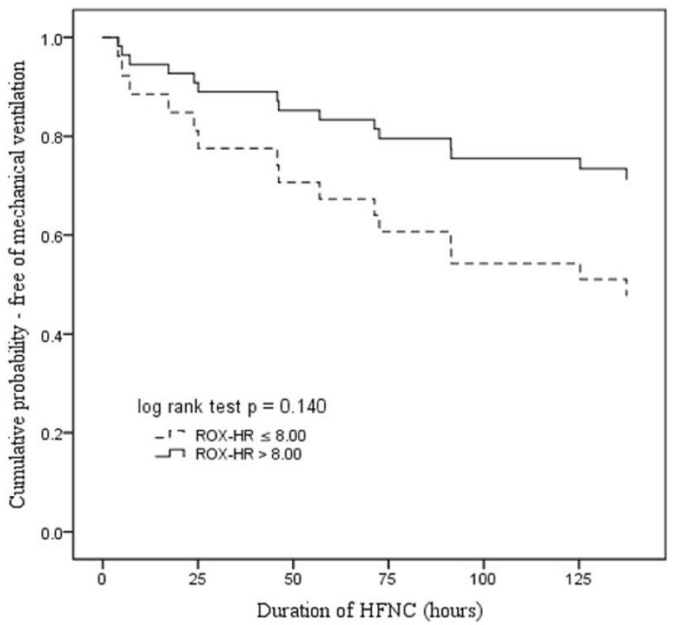

F. Porbability of HFNC success based on ROX-HR at 10 hours

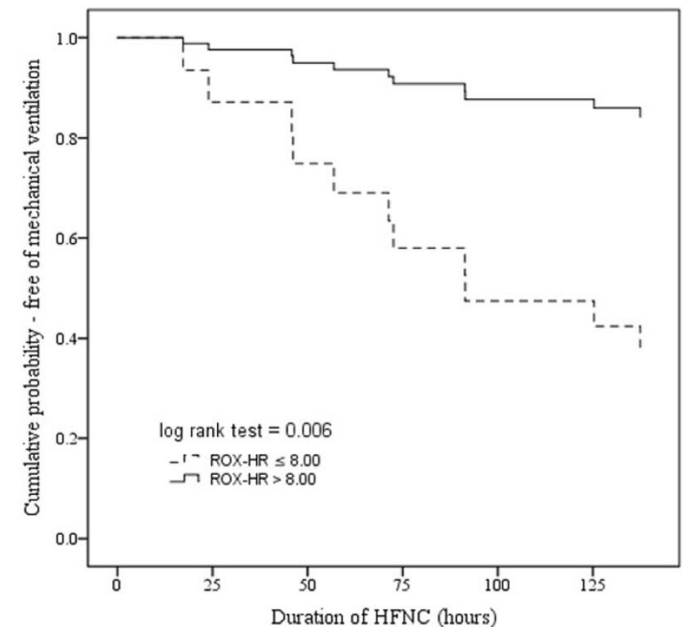

Fig. 3 a-c Kaplan-Meier plots of HFNC success probability based on ROX-HR index at 2, 6 and $10 \mathrm{~h}$ for patients initiated on HFNC for acute respiratory failure. d, e Kaplan-Meier plots of HFNC success probability based on ROX-HR index at 2, 6 and $10 \mathrm{~h}$ for patients initiated on HFNC after a planned extubation 
Table 7 Cox proportional regression analysis evaluating $\mathrm{ROX}>7.00$ and $\mathrm{ROX}-\mathrm{HR}>8.00$ for the prediction of HFNC failure in patients initiated on HFNC after a planned extubation

\begin{tabular}{|c|c|c|c|c|c|}
\hline & & Univariate analysis & $p$ value & Multivariate analysis & $p$ value \\
\hline \multirow[t]{5}{*}{$\mathrm{ROX}>7.00$} & $2 \mathrm{~h}$ & $0.519(0.184-1.459)$ & 0.213 & $0.405(0.141-1.164)$ & 0.093 \\
\hline & $6 \mathrm{~h}$ & $0.348(0.106-1.142)$ & 0.082 & $0.356(0.108-1.171)$ & 0.089 \\
\hline & $10 \mathrm{~h}$ & $0.251(0.076-0.826)$ & 0.023 & $0.287(0.083-0.998)$ & 0.050 \\
\hline & $18 \mathrm{~h}$ & $0.473(0.133-1.680)$ & 0.247 & $0.431(0.121-1.539)$ & 0.195 \\
\hline & $24 \mathrm{~h}$ & $0.169(0.035-0.814)$ & 0.027 & $0.173(0.036-0.833)$ & 0.029 \\
\hline \multirow[t]{5}{*}{$\mathrm{ROX}-\mathrm{HR}>8.00$} & $2 \mathrm{~h}$ & $0.459(0.163-1.292)$ & 0.140 & $0.483(0.171-1.364)$ & 0.169 \\
\hline & $6 \mathrm{~h}$ & $0.266(0.078-0.913)$ & 0.035 & $0.305(0.086-1.079)$ & 0.065 \\
\hline & $10 \mathrm{~h}$ & $0.176(0.051-0.604)$ & 0.006 & $0.194(0.053-0.709)$ & 0.013 \\
\hline & $18 \mathrm{~h}$ & $0.578(0.149-2.238)$ & 0.427 & $0.426(0.106-1.719)$ & 0.231 \\
\hline & $24 \mathrm{~h}$ & $0.296(0.074-1.186)$ & 0.086 & $0.303(0075-1.215)$ & 0.092 \\
\hline
\end{tabular}

Variables included in the multivariate analysis: immunocompromised host

between HFNC success and failure at all time points. Using selected cutoffs, the ROX-HR index continues to perform well in categorising patients into low and high risk for HFNC failure, as early as $2 \mathrm{~h}$ into treatment. With a cutoff of ROX $>5.80$ and ROX-HR $>6.80$, only the ROX-HR index remained significantly associated with a lower risk of HFNC failure at all time points, after correction for possible confounders. Similarly, for patients initiated on HFNC post-extubation, the ROX-HR index remained consistently lower for patients with HFNC failure. Unlike the ROX-HR index, however, the ROX index did not appear to discriminate well between patients with HFNC success vs failure at 2, 4, 8 and $18 \mathrm{~h}$.

The incorporation of the heart rate therefore appears to add value to the prediction accuracy of the ROX index. In our study, all patients with HFNC failure had a significantly higher heart rate recorded at 2 and $4 \mathrm{~h}$. In patients with HFNC initiated post-extubation, HR alone recorded at 2 and $4 \mathrm{~h}$ achieved reasonable AUROCs (0.69 and 0.70 , respectively) for the prediction of HFNC outcomes, suggesting that tachycardia, especially soon after initiation of HFNC, is associated with treatment failure. A multicentre analysis performed by Frat et al. also observed an association of HFNC failure with tachycardia, as early as $1 \mathrm{~h}$ into HFNC therapy [15]. An elevated heart rate may reflect an increased sympathetic drive or a decompensation of the cardiopulmonary system, and therefore be a marker for worse outcomes. Close to one third (31.7\%) of our study population were patients who had HFNC initiated post-extubation. In these patients, the presence of tachycardia may also reflect an impaired cardiac reserve, which is a risk factor for the development of post-extubation respiratory failure.

The ROX-HR index also provides a means of early assessment of patients on HFNC. Early prediction of HFNC failure is crucial as most patients are intubated within $24 \mathrm{~h}$ of HFNC initiation (62.3\% in our study) [14]. Furthermore, delayed intubation with HFNC has been shown to be associated with increased mortality [10, 14]. In our study, increased mortality was also seen in patients who were intubated after $24 \mathrm{~h}$. While validation in a multicentre study is needed, the ROX-HR index appears to be a promising tool for the early identification of patients at high risk of HFNC failure.

To our knowledge, our study is also the first evaluating the use of the ROX index in patients initiated on HFNC after a planned extubation. It appears that the ROX-HR performs equally well, if not better than the ROX index in these patients. Of note, the re-intubation rate seen in our cohort (35\%) is relatively higher than previous reported studies $(22-23 \%)[7,8]$. However, there are significant differences in the study populations. Our study population was relatively more ill, with a median $\mathrm{P} / \mathrm{F}$ ratio of 164, APACHE II of 15 (24 h preceding extubation) and a high proportion of immunocompromised patients (50\%). This contrasts with other studies where the reported median P/F ratios (191-240) and APACHE II (median of 11) on extubation day were relatively lower. Early recognition of the need for reintubation, which is associated with worse outcomes including mortality, is an important clinical need. Indices like the ROX-HR may therefore be a useful for early assessment during the post-extubation period.

Immunocompromised patients made up more than half our study population (56.6\%). There is a large interest in HFNC therapy for immunocompromised patients, with several studies suggesting that HFNC may be associated with reduced intubation rates [11, 18-20]. Previous studies have identified lower oxygenation and a higher organ dysfunction (SOFA scores) as predictive factors for HFNC failure in immunocompromised patients [12, 21]. However, there is still a paucity of evidence to guide the use of HFNC in immunocompromised patients. The 
substantial proportion of immunocompromised patients in our study adds strength to the applicability of the ROXHR index to these patients.

One limitation is that this was a single centre study and conducted in a medical unit, therefore excluding surgical or post-operative patients. We also did not evaluate for the presence of atrial fibrillation or the use of beta blockers in our study. It is possible that the presence of atrial fibrillation with rapid ventricular response may by itself be a poor prognostic marker for the success of HFNC. The effects of beta-blockers on the performance of the ROX-HR index are also unclear. Furthermore, bradycardia will elevate the ROX-HR index, and if associated with hemodynamic instability, will provide physicians with a false sense of assurancethis is an important consideration when applying the ROX-HR index. In our study, 2 patients had significant bradycardia $(<50$ beats $/ \mathrm{min}$ ) recorded during HFNC; both patients did not require intubation. Thirdly, evaluating a cutoff of 5.80 and 6.80, for the ROX and ROX$\mathrm{HR}$, respectively, was determined based on examination of the ROC curves of this study. Roca et al. examined the use of the ROX index for patients with acute respiratory failure from pneumonia and documented a best cutoff of 4.88 at 2,6 and $12 \mathrm{~h}[13,14]$. Applying these cutoffs for the ROX index in our study population appeared to perform with better sensitivity compared to a ROX-HR index of $>6.80$, but had poor specificity (29$51 \%$ ) for HFNC success. The ROX index $\geq 4.88$, when subjected to multivariate analysis, also did not appear to be consistently associated with HFNC outcomes.

Clearly, determining an ideal cutoff is challenging. Firstly, differences in study populations may lead to variation in findings. Compared to the report by Roca et al., a higher proportion of our patients on HFNC for acute respiratory failure was immunocompromised $(60 \%$ vs $34 \%$ ), with a higher median age (63 vs 53 years) observed in patients who failed HFNC-which may also explain the higher rate of HFNC failure seen in our study. Secondly, depending on specific clinical needs, physicians may have different priorities over the sensitivity versus specificity of the ROX or ROX-HR index, and it is likely that a "best" cutoff may also vary with differences in medical practices and ICU protocols. In our study, a lesser increase in ROX-HR index between 2 to $10 \mathrm{~h}$ and 6 to $10 \mathrm{~h}$ was also observed in patients with HFNC failure-this was not seen with the ROX index. This dynamic perspective suggests that trends in the ROX-HR index may also provide physicians with useful information. Furthermore, the absolute quantity of the index (where a ROX-HR index of $<4$ or $\geq 14$ is associated with a very high and low risk of HFNC failure, respectively) may also assist in clinical decision-making (Fig. 2 and S2). More studies are needed to evaluate these hypotheses carefully. Nevertheless, the ROX-HR index was consistently able to identify patients at high risk or low risk of HFNC failure based on identified cutoffs during the first $24 \mathrm{~h}$ into treatment. This will help to provide assurance and guidance to physicians, even in the early stages of HFNC therapy.

\section{Conclusion}

The ROX-HR index appears to be a promising tool in the early identification of patients who are at high risk of HFNC failure and for patients initiated on HFNC for acute respiratory failure as well as a preventative strategy after a planned extubation. Larger multicentre validation studies are needed to establish the role of the ROX-HR index in patients on HFNC.

\section{Supplementary information}

Supplementary information accompanies this paper at https://doi.org/10. 1186/s40560-020-00458-z.

\begin{abstract}
Additional file 1: Figure S1. Example of the ROX-HR index calculation. Figure S2. Proportion of patients with successful HFNC initiated after a planned extubation, based ROX-HR index at 2 hours (top graph) and 10 hours (bottom graph). Table S1. Comparison of the changes in ROX-HR and ROX index over different time points during HFNC. Table S2. Predic tion of HFNC failure based on a ROX and ROX-HR cut off of $<5.00$ and $<$ 6.00 for patients initiated on HFNC for acute respiratory failure and after a planned extubation, respectively. Table S3. Cox proportional regression analysis evaluating ROX $\geq 4.88$ for the likelihood of HFNC failure in patients with acute respiratory failure. Table S4. Prediction of HFNC outcomes in patients with acute respiratory failure based on ROX-HR and previously established ROX cut offs at different time points.
\end{abstract}

\section{Abbreviations}

ROX: Respiratory rate oxygenation; HFNC: High flow nasal cannula therapy; HR: Heart rate; AUROC: Area under the receiver operating curve; NIV: Noninvasive ventilation; $\mathrm{P} / \mathrm{F}$ : $\mathrm{PaO}_{2} / \mathrm{FIO}_{2}$; ICU: Intensive care unit; LPM: litres per minute; COPD: Chronic obstructive pulmonary disease; GCS: Glasgow coma scale; CCl: Charlson comorbidity index; APACHE: Acute physiology and chronic health evaluation; SOFA: Sequential organ failure assessment; CKD: Chronic kidney disease; HIV: Human immunodeficiency virus; IQR: Inter quartile range; KM: Kaplan-Meier; Cl: Confidence interval; PPV: Positive predictive value; NPV: Negative predictive value

\section{Acknowledgements}

The authors would like to thank Ms Faridah Hanim Binte Shamsuddin, Ms Constance Wei Shan Teo. and all Respiratory Therapists from the medical intensive care unit, Singapore General Hospital, for their support with patient recruitment and data collection.

\section{Authors' contributions}

K. J. G., H. Z. C. and Q. L. T. were involved in the study design and data collection. K. J. G., H. Z. C. and Q. L. T. performed the data analysis. K. J. G., H. Z. C., T. H. O., S. D. W., G. C. P. and Q. L. T. contributed to the data interpretation and writing of the manuscript. All authors reviewed the final manuscript. The authors read and approved the final manuscript.

\section{Funding}

No funding was received for this study.

Availability of data and materials

The datasets used and/or analysed during this study are available from the corresponding author on reasonable request. 


\section{Ethics approval and consent to participate}

We obtained approval from our institutional review board for this study (CIRB Ref 2016/2988). No written consent was required in view of the purely observational nature of the study.

\section{Consent for publication}

Not applicable

\section{Competing interests}

The authors have no conflict of interests to declare

\section{Author details}

'Department of Respiratory and Critical Care Medicine, Singapore General Hospital, 20 College Road, Singapore 169856, Singapore. ²Duke-National University of Singapore Graduate Medical School, Singapore, Singapore.

Received: 17 March 2020 Accepted: 10 June 2020

Published online: 22 June 2020

\section{References}

1. Frat JP, Thille AW, Mercat A, Girault C, Ragot S, Perbet S, et al. High-flow oxygen through nasal cannula in acute hypoxemic respiratory failure. $N$ Engl J Med. 2015;372:2185-96.

2. Ni YN, Luo J, Yu H, Liu D, Ni Z, Cheng J, et al. Can high-flow nasal cannula reduce the rate of endotracheal intubation in adult patients with acute respiratory failure compared with conventional oxygen therapy and noninvasive positive pressure ventilation?: A systematic review and metaanalysis. Chest. 2017;151:764-75.

3. Mauri T, Turrini C, Eronia N, Grasselli G, Volta CA, Bellani G, et al. Physiologic effects of high-flow nasal cannula in acute hypoxemic respiratory failure. Am J Respir Crit Care Med. 2017:195:1207-15.

4. Cuquemelle E, Pham T, Papon JF, Louis B, Danin PE, Brochard L. Heated and humidified high-flow oxygen therapy reduces discomfort during hypoxemic respiratory failure. Respir Care. 2012;57:1571-7.

5. Helviz Y, Einav S. A systematic review of the high-flow nasal cannula for adult patients. Crit Care. 2018;22:71.

6. Hernández G, Vaquero C, González P, Subira C, Frutos-Vivar F, Rialp G, et al. Effect of postextubation high-flow nasal cannula vs conventional oxygen therapy on reintubation in low-risk patients: a randomized clinical trial. JAMA. 2016;315:1354-61.

7. Hernández G, Vaquero C, Colinas L, Cuena R, González P, Canabal A, et al. Effect of postextubation high-flow nasal cannula vs noninvasive ventilation on reintubation and postextubation respiratory failure in high-risk patients a randomized clinical trial. JAMA. 2016:316:1565-74.

8. Maggiore SM, Idone FA, Vaschetto R, Festa R, Cataldo A, Antonicella F, et al. Nasal high-flow versus venturi mask oxygen therapy after extubation: effects on oxygenation, comfort, and clinical outcome. Am J Respir Crit Care Med. 2014;190:282-8.

9. Luo MS, Huang GJ, Wu L. High-flow nasal cannula oxygen therapy versus conventional oxygen therapy in patients after planned extubation. Crit Care. 2019;23:1-12.

10. Kang BJ, Koh Y, Lim CM, Huh JW, Baek S, Han M, et al. Failure of high-flow nasal cannula therapy may delay intubation and increase mortality. Intensive Care Med. 2015;41:623-32.

11. Azoulay E, Pickkers P, Soares M, Perner A, Rello J, Bauer PR, et al. Acute hypoxemic respiratory failure in immunocompromised patients: the Efraim multinational prospective cohort study. Intensive Care Med. 2017:43:1808-19.

12. Kim WY, Sung H, Hong SB, Lim CM, Koh Y, Huh JW. Predictors of high flow nasal cannula failure in immunocompromised patients with acute respiratory failure due to non-HIV pneumocystis pneumonia. J Thorac Dis. 2017:9:3013-22.

13. Roca O, Messika J, Caralt B, Garcia-de-Acilu M, Sztrymf B, Ricard JD, et al. Predicting success of high-flow nasal cannula in pneumonia patients with hypoxemic respiratory failure: the utility of the ROX index. J Crit Care. 2016; 35:200-5.

14. Roca O, Caralt B, Messika J, Samper M, Sztrymf B, Hernández G, et al. An index combining respiratory rate and oxygenation to predict outcome of nasal high-flow therapy. Am J Respir Crit Care Med. 2019;199:1368-76.

15. Frat JP, Ragot S, Coudroy R, Constantin JM, Girault C, Prat G, et al. Predictors of intubation in patients with acute hypoxemic respiratory failure treated with a noninvasive oxygenation strategy. Crit Care Med. 2018;46:208-15.
16. Knaus WA, Draper EA, Wagner DP, Zimmerman JE. APACHE II: a severity of disease classification system. Crit Care Med. 1985:13:818-29.

17. Vincent J-L, Moreno R, Takala J, Willatts S, De Mendonca A, Bruining H, et al. The SOFA (Sepsis-related Organ Failure Assessment) score to describe organ dysfunction/failure. Intensive Care Med. 1996;22:707-10.

18. Coudroy R, Jamet A, Petua P, Robert R, Frat JP, Thille AW. High-flow nasal cannula oxygen therapy versus noninvasive ventilation in immunocompromised patients with acute respiratory failure: an observational cohort study. Ann Intensive Care. 2016;6:45.

19. Frat JP, Ragot $S$, Girault $C$, Perbet $S$, Prat $G$, Boulain $T$, et al. Effect of noninvasive oxygenation strategies in immunocompromised patients with severe acute respiratory failure: a post-hoc analysis of a randomised trial. Lancet Respir Med. 2016:4:646-52.

20. Bin HH, Peng JM, Weng L, Liu GY, Du B. High-flow oxygen therapy in immunocompromised patients with acute respiratory failure: a review and meta-analysis. J Crit Care. 2018;43:300-5

21. Kang YS, Choi SM, Lee J, Park YS, Lee CH, Yoo CG, et al. Improved oxygenation 48 hours after high-flow nasal cannula oxygen therapy is associated with good outcome in immunocompromised patients with acute respiratory failure. J Thorac Dis. 2018;10:6606-15.

\section{Publisher's Note}

Springer Nature remains neutral with regard to jurisdictional claims in published maps and institutional affiliations.
Ready to submit your research? Choose BMC and benefit from:

- fast, convenient online submission

- thorough peer review by experienced researchers in your field

- rapid publication on acceptance

- support for research data, including large and complex data types

- gold Open Access which fosters wider collaboration and increased citations

- maximum visibility for your research: over $100 \mathrm{M}$ website views per year

At BMC, research is always in progress.

Learn more biomedcentral.com/submissions 